\title{
An Energy Efficient Sink Deployment Scheme Aiming at Extending the Lifespan for Wireless Sensor Networks
}

\author{
Deyu Lin ${ }^{1}$, Quan Wang ${ }^{2 *}$ and Jizhao Liu ${ }^{3}$ \\ ${ }^{1,2,3}$ School of Computer Science and Technology, Xidian University, No.2, South \\ Taibai Road, Yanta District, Xi'an, Shannxi, China \\ Dashing_lin@126.com,qwang@xidian.edu.cn,liujizhao2009@163.com
}

\begin{abstract}
The issue "Sink's Neighbor Problem" hinders the applications of Wireless Sensor Networks (WSNs). Owing to the heavier traffic burden they bear, the nodes close to the sink tend to dissipate their energy faster than others. They die earlier when their energy is used up. The energy efficiency is vital for WSNs because it is unpractical for the sensor nodes to be recharged. An Energy Efficient sink Deployment Scheme (EEDS) aiming at extending the lifespan for wireless sensor networks is proposed. It alleviates the energy consumption imbalance by adopting a mobile sink and regulating its route. Specifically speaking, a mobile sink moves in the monitor area with a certain rate and lingers at the fixed stops. During its move, the sensor nodes collect the data and store them in their memorizers. It is proved that the network lifetime is extended to be approximately six times compared with the static sink used only schemes via mathematical analysis. Besides, the scheme is extensible when the monitor area is enlarged. Finally, the energy efficiency of EEDS is evaluated through comparisons with the strategies only using static or mobile sinks. Experiment results show that EEDS has a good energy efficiency and can extend the network lifespan to be approximately 6 times compared with that of static-sink-used-only schemes. Besides, it is prolonged to be 148 percent of that of strategy using the mobile sink such as GMRE.
\end{abstract}

Keywords: Wireless Sensor Networks; Sink' Neighbor Problem; Network Lifespan; Mobile Sink; Energy Efficiency

\section{Introduction}

Wireless Sensor Networks (WSNs) is a network system consists of a large number of nodes capable of sensing, processing and transmitting data [1]. In spite of their constraint in process ability and storage capacity against traditional network nodes, they gain wide applications because they feature small volume and low cost [2]. Specifically, the sensor nodes are deployed in a dense way to form a network system for environment and military monitoring, medical care, endangered species tracking and safe rescue after disasters [1-3].

Most sensor nodes are powered by batteries and it is usually impossible or difficult for them to be recharged and replenished energy once being deployed. For example, in the applications such as earthquake or volcano monitoring and enemy tracking, it is unrealistic to supply energy. If some nodes run out of their energy, the network partition emerges. It leads to packet dropout of some significant monitor data and the sink cannot control the monitor areas any more. Thus the importance of energy efficiency is self-evident for WSNs. Generally speaking, the energy are mainly consumed in data sensing, processing and transmitting process. Thereinto, most of the sensor nodes' energy is used for transmission [4]. Besides, because most of WSNs adopt multi-hop transmission mode, the nodes close to the sink bear a heavier energy burden. It results in energy imbalance and this issue is known as "Hot Spot Problem"or "Sink Neighbor 
Problem" [5-7]. Therefore, it is vital to achieve energy balance. As the energy is mainly consumed in the communication module, the primary methods to extend the network lifetime as is to cut down the energy depletion in transmission and to balance the energy consumption.

The remainders of the paper are organized as follows: The next section discusses the related works. The energy consumption model and some assumptions are proposed in section 3. Section 4 presents the mathematical analysis about the energy benefit resulted from the mobile sink firstly. Subsequently An Energy Efficient sink Deployment Scheme (EEDS) aiming at extending the lifespan is proposed. Section 5 evaluates the energy efficiency of the EEDS through extensive simulation experiments. Section 6 gives the conclusions and discuss about the paper.

\section{Related Works}

Recent years have witnessed a series of research products concerning the energy efficiency. To relief the energy gloom caused by the "Hot Spot Problem", the node mobility is taken into consideration [2-10]. Mobile relay [12-13] and mobile sink [3,10] are regulated to move along a certain or random path periodically. Thus the energy balance is achieved because of the "Hot Spot" changing from time to time. Ref. [13] firstly proposes that the multi-hop transmission can be converted to one-hop communication via utilizing mobile relays. Although the energy consumption is reduced effectively, it is at the cost of the network delay. The nodes store the data till the mobile relay arrives in their communication range. Then they send the useful messages they sensed to the relay immediately. Although energy is saved, it is obviously inappropriate for the real time applications. Refs. [5-8] translate the mobile sink problem into linear programming model. The initial energy of each node as well as traffic flow reservation are regarded as restrictions to maximize the network lifespan. Ref. [12] supposes that the mobile sink lingers at fixed stop. The sojourn time is regarded as the variable and the network lifetime is defined as the sum of the sojourn time spending on different stops. Finally the maximum lifetime is obtained subject to the given initial energy. Although linear programming is able to derive the optimal solution, it should not be ignored that it is rather complicated to control. The complexity is derived from the fact that the whole network topology need to be considered in order to determine the optimal sojourn time of mobile sink at each stop. Meanwhile, every time when it lingers, the Mobile Sink should broadcast its position to others. All these processing overheads increase the energy consumption.

The contributions of the paper are as follow: An energy efficient sink deployment scheme is proposed. In the strategy, one Mobile Sink is initially deployed in the center of the monitor area. Besides, it moves rapidly along a certain path. In additional, to receive data with a small delay, seven fixed stops are defined. Whenever the Mobile Sink reach the stop, it lingers to receive the data from nodes within its transmission range. Compared with the existent mobile sink schemes, the network control is much simpler and the network topology can be extended. Furthermore, the energy imbalance caused by the "Hot Spot Problem" is alleviated compared with those using the static sink only.

\section{Energy Consumption Model and Relative Assumptions}

\subsection{Energy Consumption Model}

The energy consumption model adopted in this paper is the same as that in Refs.[2, $[4-5,14]$. It is called the First Older Radio Model and is specifically denoted as following expressions (1) and (2):

$$
e_{t x}=E_{\text {elec }}+\varepsilon_{\text {amp }} d^{\alpha}
$$




$$
e_{r x}=E_{\text {elec }}
$$

Where $e_{r x}$ and $e_{t x}$ represent the energy for each sensor node to receive and transmit per bit message respectively. $E_{\text {elec }}$ and $\varepsilon_{\text {amp }}$ denote the energy consumption for transmitter circuit and transmitter amplifier respectively, and $\alpha(2 \leq \alpha \leq 4)$ is the propagation loss exponent. In detail, $\alpha$ is 2 for free space and increases to be 4 when obstacles exist. Generically, $\alpha$ is set to be range from 2 to 4 . In the paper it is set to be 2 as the nodes' transmission range is under controlled to cut down the energy consumption further.

\subsection{Relative Assumptions}

To facilitate our further exposition, some assumptions and notations are put forward below:

All the nodes transmit data with fixed radius and they are uniformly distributed. The node density is denoted as $\rho$ and the network topology is shown as Figure 1.

Each node generates data with the same rate and can either act as source or relay node.

All the sensor nodes keep stationary and possess the same initial energy which is denoted as $E_{\text {initial }}$.

The sink has no limit in processing, storing capacity and has infinite energy supply compared with the common sensor nodes.

The Mobile Sink (M-Sink) moves at rapid speed and the data rate is relatively low, so the delay resulted from route table reconstruction can be ignored.

The set of sensor nodes is denoted as $N$ and for any node $i, j \in N$, the distance between them is represented as $d(i, j)$.

\section{An Energy Efficient Sink Deployment Strategy (EEDS) aiming at extending the Lifespan}

\subsection{Mathematical Model}

Lemma: The network lifespan when adopting EEDS is extended to be approximately 6 times compared with that using static sink.

Proof: The discussion is about the average energy consumption of the nodes close to the sink when only Static Sink (Ssink) is adopted. According to the assumptions presented in section 3.2, all the nodes in the circle area with its center at the sink and of radius $\mathrm{R}$ transmit their data collected to the Sink via one-hop fashion. The set containing these nodes is denoted as $N_{\text {Sink }}$ and $N_{\text {Sink }}=\{i \mid d(i$, Sink $) \leq R, i \in N\}$. Any node $i \in N_{\text {Sink }}$ not only generates its own message but also receives and processes the data from the outer areas. These nodes belong to the "Hot Spot Area" and they bear heavier traffic load. When there is only one Static Sink, the routing fashion is hop-by-hop. Therefore the nodes in $N_{\text {Sink }}$ bear heavier energy burden. Thus they tend to use up their energy faster than the nodes in outer areas. Since the network lifespan is evaluated by the time till the first node dies in most applications. Therefore the network lifetime can be represented by that of the nodes in $N_{\text {Sink }}$. For the sake of simplicity, it is assumed that each node generates one unit data per unit of time.The following analysis shows the total receiving energy $E_{r}$ and transmitting energy $E_{t}$ of nodes in set $N_{\text {Sink }}$. From Figure 1, the area of shade $A_{\text {shade }}$ can be obtained as follow: 


$$
A_{\text {shade }}=2\left(\pi \cdot R^{2}-\sqrt{3} / 4 \cdot R^{2}\right)=0.18 R^{2} .
$$

Besides, the values of $E_{r}$ and $E_{t}$ are as follow:

$$
\begin{gathered}
E_{r}=E_{\text {elec }}(6 \cdot \pi-12 \cdot 0.18) \rho \cdot R^{2}, \\
E_{t}=\left(E_{\text {elec }}+\frac{1}{4} R^{2}\right)(7 \cdot \pi-12 \cdot 0.18) \rho \cdot R^{2} .
\end{gathered}
$$

Therefore the average energy consumption $\bar{E}_{N_{\text {Sink }}}$ for the nodes in $N_{\text {Sink }}=\{i \mid d(i$, Sink $) \leq R, i \in N\}$ is :

$$
\begin{aligned}
& \bar{E}_{N_{\text {Sink }}}=\frac{E_{r}+E_{t}}{\rho \int_{0}^{2 \pi} \int_{0}^{R} r d \theta d r}=\frac{E_{\text {elec }}(6 \pi-12 \cdot 0.18) \rho R^{2}+\left(E_{\text {elec }}+\frac{1}{4} R^{2}\right)(7 \pi-12 \cdot 0.18) \rho R^{2}}{\rho \pi R^{2}} \\
& =11.62 E_{\text {elec }}+2.01 R^{2}
\end{aligned}
$$

Thus the network lifespan $T_{\text {network }}$ is :

$$
T_{\text {network }}=\frac{E_{\text {initial }}}{\overline{E_{N_{\text {Sink }}}}}=\frac{E_{\text {initial }}}{11.62 E_{\text {elec }}+2.01 R^{2}}
$$

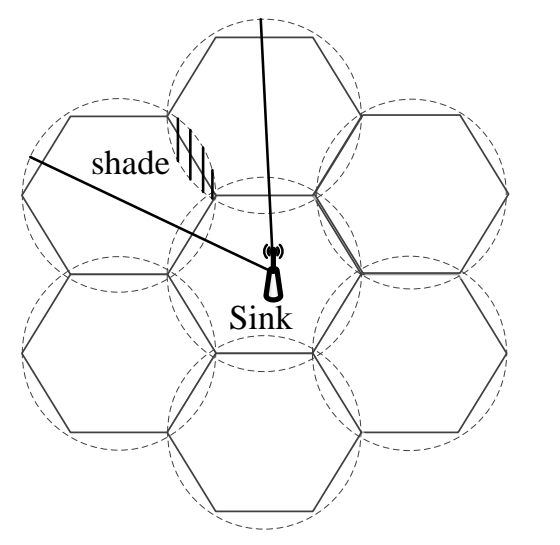

Figure 1. The Network Topology of Sink Strategy

When the EEDS is adopted, the Mobile Sink initially locates at the center of the network area and it moves at rapid rate along the path marked in blue dotted lines in Figure 2. According to the assumptions given in section 3.2, The Mobile Sink (M-Sink) moves at a rapid speed and the data rate is relatively low, so the delay resulted from route table reconstruction can be ignored. Whenever the Mobile Sink reaches a stop, the nodes with a certain distance from it send out their sensed data. Owing to the move of the M-Sink, all the data transmission follows the one-hop fashion. The nodes which are $\mathrm{R}$ away from the Mobile Sink is denoted as $N_{M-\operatorname{Sink}}$ and $: N_{M-\operatorname{Sink}}=\{i \mid d(i, M-\operatorname{Sink}) \leq R, i \in N\}$. Thus the energy consumption for reception $E_{r}^{\prime}$ of $N_{M-\operatorname{sink}}$ is :

$$
E_{r}{ }^{\prime}=\rho E_{\text {elec }}\left[\int_{0}^{\pi} \int_{0}^{R} r d{ }_{r} d \theta=\pi \rho R^{2} E_{\text {elec }} .\right.
$$

Likewise, $E_{t}$ is: 


$$
E_{t}^{\prime}=\left(E_{\text {elec }}+1 / 4 R^{2}\right) \rho \int_{0}^{2 \pi} \int_{0}^{R} r d r d \theta=E_{\text {elec }} \cdot \rho \pi R^{2}+\frac{1}{4} \pi \rho R^{4}
$$

The average energy consumption $\bar{E}_{N-M \sin k}$ in set $N_{M-\sin k}$ is :

$$
\bar{E}_{N-M \sin k}=\frac{E_{r}+E_{t}}{\rho \int_{0}^{\pi} \int_{0}^{R} r d r d \theta}=2 E_{\text {elec }}+0.25 R^{2} .
$$

Then the network lifespan is :

$$
T_{\text {Network }}=\frac{E_{\text {initial }}}{\bar{E}_{N-M \sin k}}=\frac{E_{\text {initial }}}{2 E_{\text {elec }}+0.25 R^{2}} .
$$

Obviously, from expressions(6) and (10) the following is obtained:

$T_{\text {Network }}=5.81 T_{\text {Network }}$

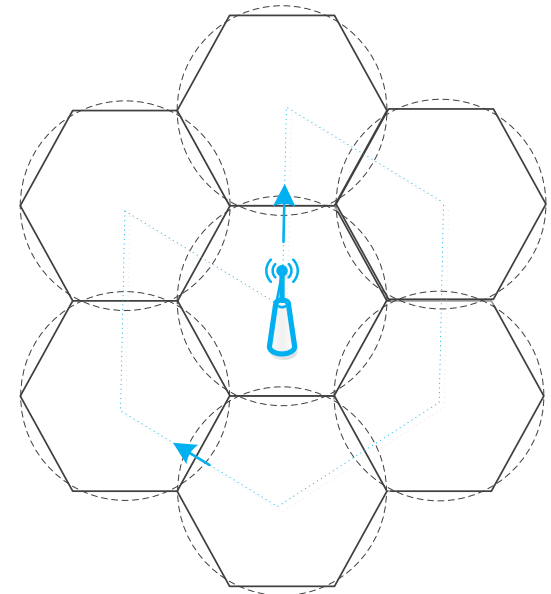

Figure 2. The Network Topology with Mobile Sink

\subsection{An Energy Efficient Sink Deployment Scheme (EEDS) aims at extending the Lifespan}

According to Section 4.1, the Energy Efficient sink Deployment Scheme (EEDS) aiming at extending the lifespan is obtained. The specific algorithm is described as follow.

The Mobile Sink (M-Sink) is utilized in the EEDS. M-Sink initially locates at the center of the network topology and moves along the specific path marked in blue dotted line shown in Figure 2. Each point of inflexion is the station of the M-Sink. Whenever the Sink reaches a station, it lingers and broadcasts to the nodes within its transmission range. Then it waits for the messages from the nodes lying in its transmission range. The length of sojourn time is determined as follow: if the M-Sink has not received any data for a while, it broadcasts a Request Message to affirm weather to wait or not. The nodes with data to in its memory send a reply back on receiving the Request Message. Otherwise they discard the Request Message simply. If the M-Sink has not received any replies, it moves to the next station. All the nodes beyond the influence of the M-Sink store the data sensed till it arrives at the corresponding influence areas.

The EEDS scheme can be also extended when the monitor area is enlarged as the Figure 3 shows. One more Mobile Sink is adopted and it moves along a path near the boundary of the monitor area. Just Figure 3 shows, two Mobile Sinks work together to balance the energy consumption and extend the lifespan of WSNs. 


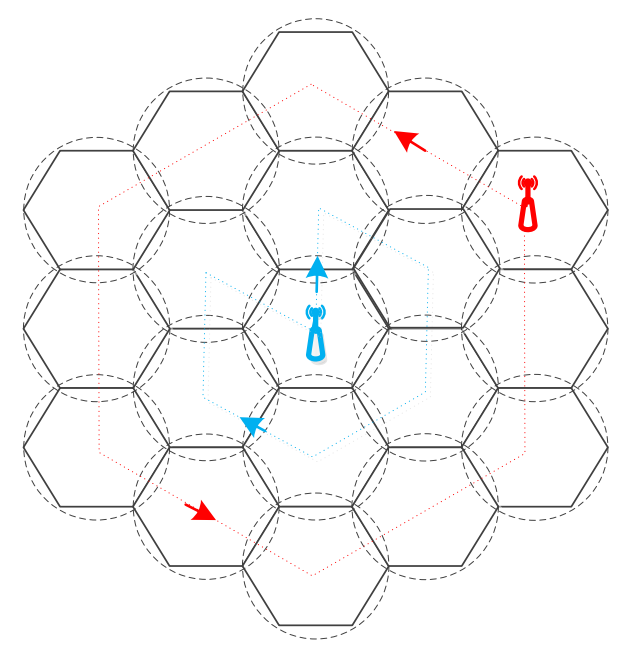

Figure 3. The Expansion of Scheme EEDS

\section{Experiment and Results Analysis}

\subsection{Evaluation Parameters}

The simulation experiment is conducted via NS2. A hundred sensor nodes are uniformly distributed in the circle area with the radius of $l=260$. The initial energy of each node $E_{\text {initial }}$ is $2 \mathrm{~J}$. The energy consumption for the circuit to send or receive one bit message is $50 \mathrm{~nJ}$. The value of $\varepsilon_{\text {amp }}$ is $13 \mathrm{pJ} / \mathrm{bit} / \mathrm{m}^{2}$. Finally, the speed of M-Sink is set to be $50 \mathrm{~m} / \mathrm{s}$.

To evaluate the energy efficiency of EEDS, several performance metrics are defined as follow.

The curve of the number of the nodes alive. It reflects the varies of the quantity of the nodes alive. This parameter can reflect the network lifespan intuitively.

The time till the first node dies. It means the time when the first node exhausts its energy. For simplicity, it is denoted as FND.

The energy efficiency. It denotes the data received by the sink given a certain amount of energy consumed. It perfectly reflect the performance of the scheme EEDS.

Finally the simulation results are compared with that of Static sink strategy(Ssink) and the Mobile Sink strategy. In the paper the Mobile Sink strategy GMRE is compared against EEDS due to its high energy efficiency. Ref[5] shows that it extends the network lifetime to be four times as long as that of Ssink.

\subsection{Results Analysis}

Figure 4 shows the trends of the number of nodes alive when Ssink, EEDS and GMRE are adopted respectively. The Ssink forwards data in the multi-hop fashion. Metrics FND of the three strategies are 365, 1380 and 2040 respectively as showed in Figure 4. According to section 5.1, the conclusion that the network lifespan of EEDS is 5.59 times as long as that of Ssink and is 0.48 times longer than that of GMRE is drawn. It means that the network lifespan can be extended to be approximately six times as long as that of Ssink. Besides, the energy efficiency is improved by fifty percent over GRME. It complies with the lemma of section 3.1. Because the overhead of routing table reconstruction caused by the M-sink's movement is ignored in the theoretical proof, the simulation results is a little less than the theoretical value. 


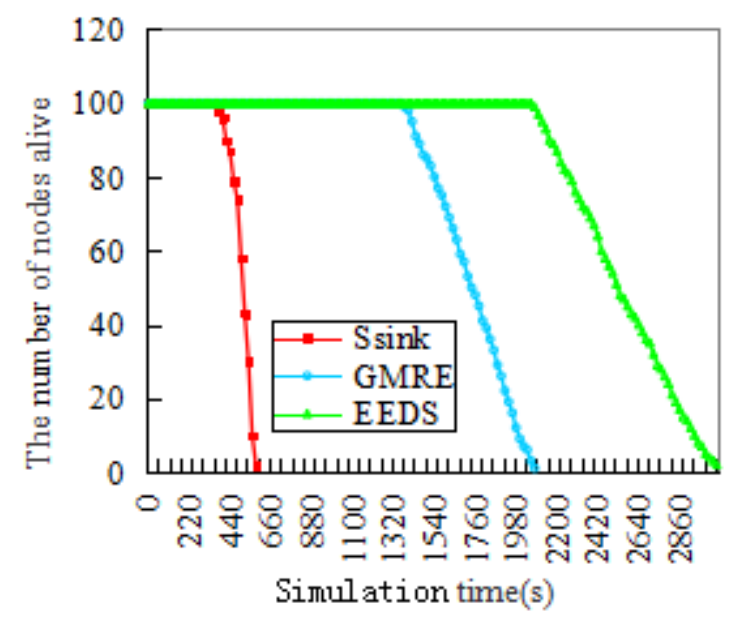

Figure 4. The Number of Nodes Alive

Figure 5 shows the energy consumed varies with the simulation time. At the initial moment, the total energy of the network is $100 * 2 J$. As time goes by, Ssink depletes energy fastest. It can be easily obtained from figure 5 owing to the Ssink's large slope. It is also obvious that EEDS consumes the energy slowest. Using strategy Ssink, the "Hot Spot Problem" cannot be solved effectively, thus the nodes in the "Hot Spot" drained away earlier than others. The problem is alleviated via the mobile sink's movement in GMRE, but the energy of some nodes still dissipates quickly due to the multi-hop transmission. In the paper, the strategy regulating the path of mobile sink reduces the energy consumption resulted from the "Hot Spot Problem". Moreover, most of the data is transmitted via one-hop fashion, thus the energy is consumed slowest and the network lifespan is accordingly lengthened.

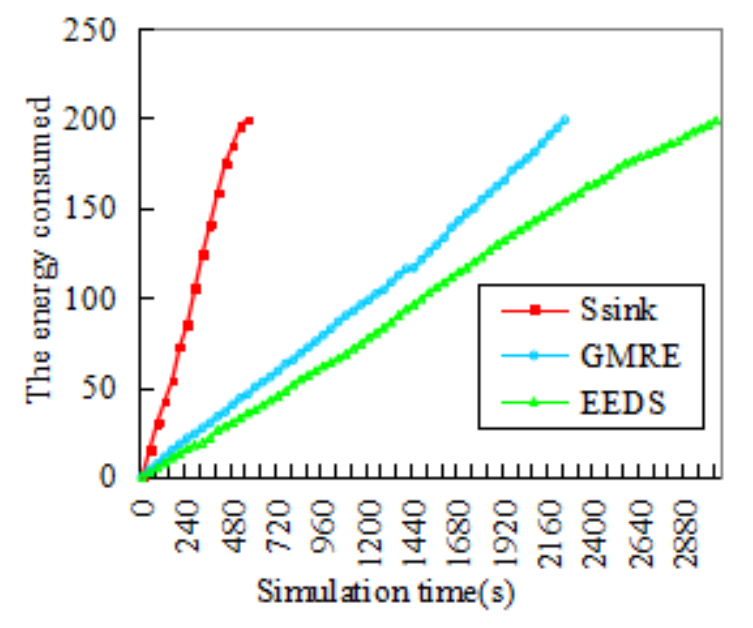

Figure 5. The Sum of Energy Consumed

Figure 6 shows the average value of data received by the sink per unit energy consumed. It indicates that the value of Ssink is mimimum and that of EEDS is maxmum. It means that the energy efficiency of EEDS is the highest compared with other two schemes. As the EEDS regulates the path of the mobile sink, all the nodes transmit their data in one-hop fashion, thus its energy efficiency is improved. 


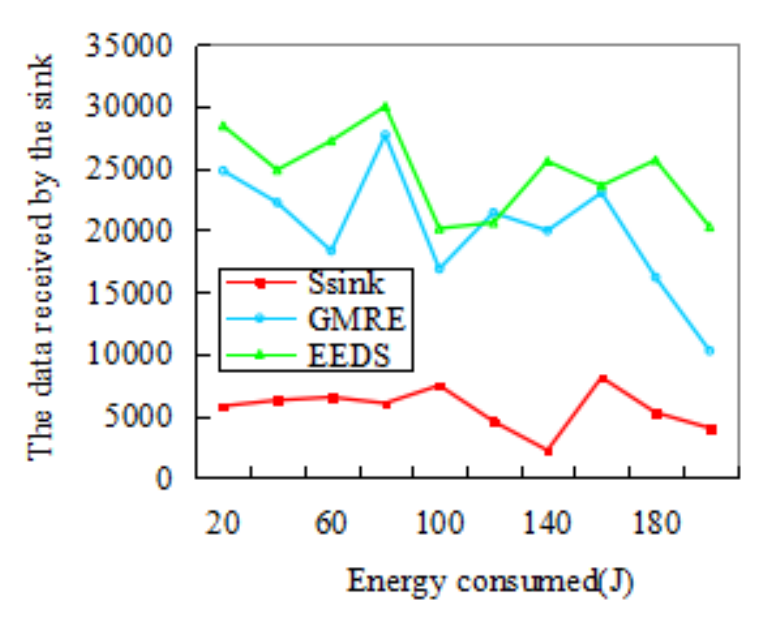

Figure 6. The Data Received by the Sinks

\section{Conclusions and Discussions}

The energy constraint is one of the inherent problems of WSNs. The "Hot Spot Problem" caused by multi-hop transmission makes things worse. The paper proposed a novel strategy EEDS to solve the problem. The theoretical analysis is presented first and the conclusion that the network lifetime is effectively extended to be six times is drawn. Subsequently the Energy Efficient sink Deployment Scheme (EEDS) aiming at extending the lifespan for WSN is proposed. Finally extensive simulation experiments are conducted. The experiment results confirm that the lifespan of WSNs is lengthened to be 5.59 times as long as that of Ssink and meanwhile it is 0.48 times longer than that of GMRE.

The research work and the results of this paper can be applied to the application scene where the event data rate is relatively low. Meanwhile, the EEDS can also be expanded to the larger monitor area if only some more Mobile Sinks are utilized to collect data.

\section{Acknowledgments}

The authors would like to thank the reviewers for their detailed reviews and constructive comments, which have helped improve the quality of this paper. The paper is supported by the National Natural Science Foundation of China (Program ID 61572385).

\section{References}

[1] I. F. Akyildiz, W. S. Sankarasubramaniam and E. Y. Cayirci, "A Survey on Sensor Networks", Communications Magazine, vol. 40, no. 8, (2002).

[2] W. R. Heinzalman, A. Chandrakasan and H. Balakrishnan, "Energy-efficient Communication Protocol for Wireless Microsensor Networks", Proceedings of the 33rd Hawaii International Conference on System Sciences, Hawaii, USA, (2000).

[3] S. Basagni, A. Carosi, E. Melachrioudis, C. Petrioli and Z. M. Wang, "Controlled Sink Mobility for Prolonging Wireless Sensor Networks Lifetime", Wireless Networks, vol. 14, no. 6, (2007).

[4] V. Zoltan, V. Rolland and V. Attila, "Deploying Multiple Sinks in Multi-hop Wireless Sensor Networks", IEEE International Conference on Pervasive Services, Istanbul, (2007).

[5] S. Basagni, A. Carosi, E. Melachrinoudis, C. Petrioli and Z. M. Wang, "A New MILP Formulation and Distributed Protocols for Wireless Sensor Networks Lifetime Maximization”, IEEE International Conference on Communications, (2006).

[6] W. Liang, J. Luo and X. Xu, "Prolonging Network Lifetime via a Controlled Mobile Sink in Wireless Sensor Networks", Global Telecommunications Conference, (2010).

[7] W. Wang, S. Vikram and K. C. Chua, "Extending the Lifetime of Wireless Sensor Networks through Mobile Relays", IEEE/ACM Transcations on Networking, vol. 16, no. 5, (2008).

[8] Y. Yun and Y. Xia, "Maximizing the Lifetime of Wireless Sensor Networks with Mobile Sink in Delay-tolerant Applications", IEEE transactions on mobile computing, vol. 9, no. 9, (2010).

[9] S. Rahim, H. Rahim, R. D. Hhan, M. Z. Siddiqi, M. A. Qureshi, U. Qasim, Z. A. Khan, S. Mahmood and 
N. Javaid, "Circular Joint Sink Mobility Scheme for Wireless Sensor Networks", Advanced Information Networking and Applications Workshops, (2015).

[10] L. Cheng, C. Chen and J. Ma, "Selection Scheme of Mobile Sinks in Wireless Sensor Networks", Journal on Communications, vol. 29, no. 11, (2008).

[11] Y. Shi and Y. T. Hou, "Theoretical Results on Base Station Movement Problem for Sensor Network", the IEEE INFOCOM 2008 Proceedings, (2008).

[12] S. R. Gandham, M. Dawande, R. Prahash and S. Venkatesan, "Energy Efficient Schemes for Wireless Sensor Networks with Multiple Mobile Base Stations", Global Telecommunications Conferences, (2003).

[13] R. C. Shah, S. Roy, S. Jain and W. Brunette, "Data MuLEs: Modeling and Analysis of a Three-tier Architecture for Sparse Sensor Networks", Sensor Network Protocols and Applications, vol. 1, (2003), pp. 2-3.

[14] D. Lin, Q. Wang, D. Lin and Y. Deng, "An Energy-efficient Clustering Routing Protocol Based on Evolutionary Game Theory in Wireless Sensor Networks", International Journal of Distributed Sensor Networks, (2015).

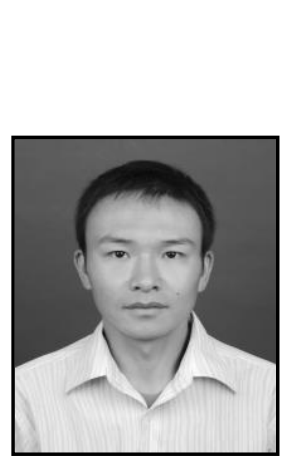

\section{Authors}

Deyu Lin, he received the B.S. degree in Computer Science and Technology from Xianyang Normal University in 2011 and B.S. degree in Computer Application Technology from Shandong University of Science and Technology in 2013 respectively. Lin is currently studying for his doctor in computer system architecture in Xidian University. His research interests are WSNs, Ad Hoc network.

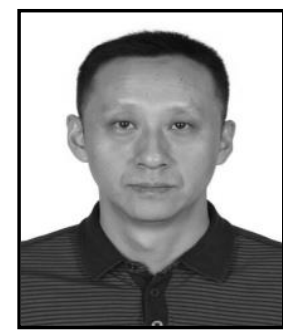

Quan Wang, he received the B.S., M.S. and Ph.D. degrees in Computer Science from Xidian University in 1992, 1997 and 2008 respectively. He is now a Professor at the School of Computer Science and Technology and the institute director of the Institute of Computer Peripheral Equipment. His research interest includes embedded system, 3-D printing and wireless networks.

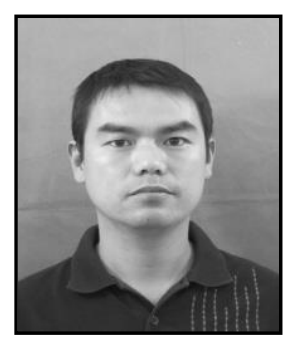

Jizhao Liu, he received the B.S. degree in Computer Application Technology from China University of Mining and Technology in 2002 and B.S. degree in Computer Application Technology from Henan University of Technology in 2009 respectively. Lin is currently studying for his doctor in computer system architecture in Xidian University. His research interests are WSNs, Ad Hoc network. 
International Journal of Future Generation Communication and Networking Vol. 9, No.8, (2016) 